\title{
GPS/INS Integrated Navigation Systems based on Modified Gaussian Sum Filtering Methods
}

\author{
T. Sato, H. Okuno, Y. Kubo, and S. Sugimoto \\ Department of Electrical and Electronic Engineering, Ritsumeikan University
}

\begin{abstract}
In this paper, we propose a modified Gaussian Sum filtering method and apply it to land-vehicle INS(Inertial Navigation System)/GPS(Global Positioning System) integrated navigation as well as In-Motion Alignment systems. Then we show the experimental results of INS/GPS integrated system by using simulated data, with assuming the various error sources such as the errors of GPS measurement, INS sensors and various running trajectories.
\end{abstract}

\section{INTRODUCTION}

In the conventional INS navigation, the initialization of the INS navigation states such as position, velocity and attitude must be completed prior to vehicle motion. In general, the initialization require the vehicle to stop at the initial position for at least 5 to 10 minutes. It is, however, inconvenient and impractical in case that there is not enough time to stop at the initial position. Therefore In-Motion Alignment algorithm that enables the vehicle to start instantly is motivated.

For the INS error model in our proposed algorithm, the linear large azimuth error model [1] is adopted with some modifications. The large azimuth error model is not linearized.

In this work, we apply the nonlinear filters: Gaussian Sum filter (GSF) [2], Unscented Kalman filter (UKF) [3] and modified Gaussian Sum filter, to the In-Motion Alignment. Firstly, we briefly review the algorithms of these filters. Then, we show the simulation results of some vehicle trajectories, and discuss the feasibility of the nonlinear filters to the In-Motion alignment.

\section{DESCRIPTION OF THE SYSTEM}

In this work, closed-loop mechanization is adopted for the GPS/INS integration. Fig. 1 shows the architecture of the integration with major data paths between the system components. The components of the system are strapdown INS and GPS receiver.

The INS contains accelerometer and gyro. Based on the measured acceleration and angular rate, the INS computes the position, velocity and attitude of the vehicle relative to their initial value at high frequency. But there exist unbounded position errors that grow slowly with time. The position errors grow 1 to 2 nautical miles per hour with high performance Ring Laser Gyro (RLG). The concept of the integrated navigation system of Fig. 1 is to reduce the INS errors by using some external measurement of the position and/or velocity etc. In this research, GPS double differenced pseudorange and carrier phase measurements are employed as external measurements to remove the INS errors. The nonlinear filter estimates the errors in the navigation and attitude information using the raw GPS data.

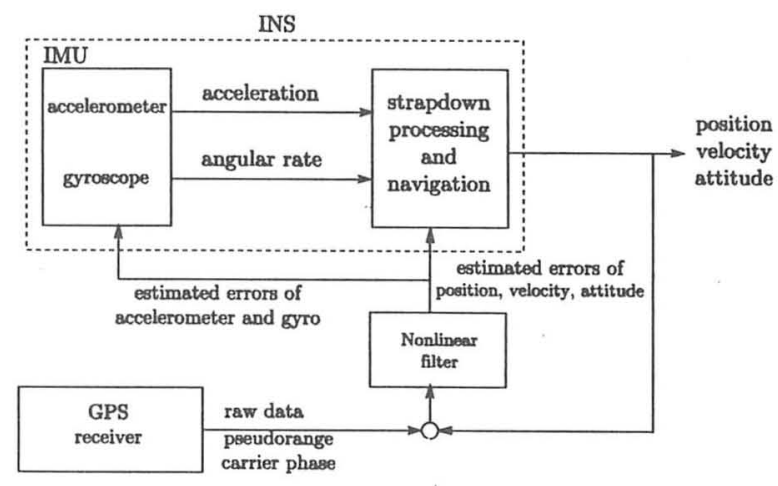

Fig. 1: Description of the system

\section{COORDINATE SYSTEMS}

To integrate the navigation systems, it is important to consider the coordinate systems that the navigation systems or included sensors refer to. This section defines the coordinate frames used in this paper and represents the angular relationship between them. The coordinate frames are defined as follows: 
i) The $E$ frame $\left(X_{E}, Y_{E}, Z_{E}\right)$ is the right-handed earth fixed coordinate frame. It has the origin at the center of the earth; the $Z_{E}$-axis is directed toward the North Pole; the $X_{E^{-}}$and $Y_{E}$-axes are located in the equatorial plane, whereby the $X_{E}$-axis is directed toward the Greenwich Meridian. It is used for the definition of position location such as latitude and longitude.

ii) The $L$ frame $\left(X_{L}, Y_{L}, Z_{L}\right)$ is the right-handed locally level coordinate frame. The $X_{L^{-}}$and $Y_{L}$-axes are directed toward local north and east respectively; $Z_{L}$-axis is downward vertical at the local earth surface referenced position location. It is used for defining the angular orientation of the local vertical in the $E$ frame.

iii) The $C$ frame $\left(X_{C}, Y_{C}, Z_{C}\right)$ is the right-handed computer frame that is defined by rotating the $L$ frame around negative $Z_{L}$-axis by the "wander angle" $\alpha$, the $Y_{C}$-axis is directed toward the negative $Y_{L}$-axis and the $Z_{C}$-axis is directed toward the negative $Z_{L}$-axis (upward vertical). It is used for integrating acceleration into velocity, and used as the reference for describing the strapdown sensor coordinate frame orientation.

iv) The $B$ frame $\left(X_{B}, Y_{B}, Z_{B}\right)$ is the strapdown inertial sensor coordinate frame (body frame). The $X_{B}$-axis is directed toward the head of the vehicle; the $Y_{B}$-axis is the right-hand of the vehicle; the $Z_{B}$-axis is downward vertical to the $X_{B}-Y_{B}$ plane. The frame is fixed on the vehicle and rotates with the motion of the vehicle.

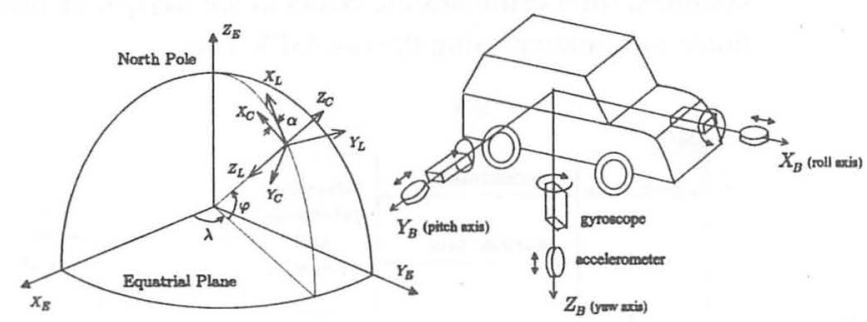

Fig. 2: The $E, L, C, B$ frame and sensors

Fig. 2 shows the spatial image of the $E, L, C$ and $B$ frames, where $\lambda$ and $\varphi$ represent the geodetic longitude and the latitude respectively.

In inertial computations, three components of acceleration sensed with respect to the $B$ frame have to be transformed onto the $C$ frame. The velocity and position of the vehicle are then computed with respect to the $C$ frame. Such a transformation is known as an Euler angle transformation. Then the coordinates $\left(x_{B}, y_{B}, z_{B}\right)$ in the $B$ frame are transformed into $\left(x_{C}, y_{C}, z_{C}\right)$ in the $C$ frame as follows:

$$
\left[\begin{array}{l}
x_{C} \\
y_{C} \\
z_{C}
\end{array}\right]=T_{B}^{C}\left[\begin{array}{l}
x_{B} \\
y_{B} \\
z_{B}
\end{array}\right]
$$

where, $T_{B}^{C}$ is the direction cosine matrix.

\section{INS ERROR MODEL}

The direction cosine matrix $T_{E}^{C}$ is described as follows

$$
T_{E}^{C}=T_{L}^{C} T_{E}^{L} .
$$

The computed matrices $\overline{T_{L}^{C}}$ and $\overline{T_{E}^{L}}$ contain errors $\delta T_{L}^{C}$ and $\delta T_{E}^{L}$, respectively, such that the error $\delta T_{E}^{C}$ is described by

$$
\begin{aligned}
\delta T_{E}^{C} & \equiv \overline{T_{E}^{C}}-T_{E}^{C} \\
& =\overline{T_{L}^{C}} \overline{T_{E}^{L}}-T_{L}^{C} T_{E}^{L} \\
& =\left\{\delta T_{L}^{C}\left[I-\left(\delta r_{L} \times\right)\right]-T_{L}^{C}\left(\delta r_{L} \times\right)\right\} T_{E}^{L} \\
& =\mathcal{R} T_{E}^{L},
\end{aligned}
$$

where $\delta \boldsymbol{r}_{L} \equiv\left[\delta r_{L, x}, \delta r_{L, y}, 0\right]^{\mathrm{T}}$ is horizontal angular position error and we can show the relation $\overline{T_{E}^{L}}=[I-$ $\left.\left(\delta r_{L} \times\right)\right] T_{E}^{L}$ with assumption that $\delta r_{L, x}, \delta r_{L, y}$ are small. Also, $(a \times)$ for $3 \times 1$ vector $a=\left[a_{x}, a_{y}, a_{z}\right]^{\mathrm{T}}$ is the skewsymmetric matrix defined by

$$
(a \times) \equiv\left[\begin{array}{ccc}
0 & -a_{z} & a_{y} \\
a_{z} & 0 & -a_{x} \\
-a_{y} & a_{x} & 0
\end{array}\right]
$$

And $\mathcal{R}$, the position error matrix [1], is defined as follows

$$
\mathcal{R} \equiv\left[\begin{array}{ccc}
\delta \cos \alpha & -\delta \sin \alpha & \delta r_{C, y} \cos \delta \alpha-\delta r_{C, x} \sin \delta \alpha \\
-\delta \sin \alpha & -\delta \cos \alpha & -\delta r_{C, x} \cos \delta \alpha-\delta r_{C, y} \sin \delta \alpha \\
-\delta r_{L, y} & \delta r_{L, x} & 0
\end{array}\right]
$$

where $\delta \sin \alpha \equiv \sin (\alpha+\delta \alpha)-\sin \alpha, \delta \cos \alpha \equiv \cos (\alpha+$ $\delta \alpha)-\cos \alpha$. According to [1], we have

$$
\dot{\mathcal{R}}=\mathcal{R}\left(\omega_{E / L}^{L} \times\right)-\left(\overline{\omega_{E / C}^{C}} \times\right)\left(T_{L}^{C}+\mathcal{R}\right)+\left(\omega_{E / C}^{C} \times\right) T_{L}^{C},(
$$

where the dot above a letter denotes differentiation with respect to time, the vector $\omega_{E / L}^{L}$ is the rotation rate of the $L$ frame with respect to the $E$ frame in the $L$ frame coordinate system and also the vector $\omega_{E / C}^{C}$ is similarly denoted.

Eq. (6) gives the position error $\left(\delta r_{C, x}, \delta r_{C, y}\right)$ equations in the INS navigation, and also gives the relations of state variables: the wander angle error $\delta \alpha$.

\subsection{Velocity Error Model}

The computed velocity $\overline{v_{C}}$ also contains the velocity error $\delta v_{C}$, and the velocity equation is as follows:

$$
\dot{v}_{C}=f_{C}-\left(\rho_{C}+2 \Omega_{C}\right) \times v_{C}+g_{C},
$$

where $f$ is the no gravitational specific force vector, $\rho$ is relative rate vector, and $\Omega$ is earth rate vector. The specific force is proportional to the inertial acceleration of the system due to all forces except gravity measured by the accelerometer. $g_{C}$ is the gravity vector considered positive toward the 
center of the earth in the $C$ frame. From Eq. (7), the velocity $\operatorname{error}\left(\delta v_{C, x}, \delta v_{C, y}, \delta v_{C, z}\right)$ equation is described as

$$
\begin{gathered}
\delta \dot{\boldsymbol{v}}_{C}=\boldsymbol{b}_{C}+\boldsymbol{f}_{C} \times \delta \boldsymbol{\theta}_{C}+\boldsymbol{v}_{C} \times\left(\delta \boldsymbol{\rho}_{C}+2 \delta \boldsymbol{\Omega}_{C}\right)- \\
\left(\boldsymbol{\rho}_{C}+2 \boldsymbol{\Omega}_{C}\right) \times \delta \boldsymbol{v}_{C}-\left(\delta \boldsymbol{\rho}_{C}+2 \delta \boldsymbol{\Omega}_{C}\right) \times \delta \boldsymbol{v}_{C}+\delta \boldsymbol{g}_{C},(8)
\end{gathered}
$$

where $\delta \theta_{C} \equiv\left[\delta \theta_{C, x}, \delta \theta_{C, y}, \delta \theta_{C, z}\right]^{\mathrm{T}}$ is the attitude error.

\subsection{Attitude Error Model}

The attitude error is defined by tilts of vehicle. The error is associated with the transformation matrix error $\delta T_{B}^{C}$ caused by the attitude error, $\delta \boldsymbol{\theta}_{C}$. The computed matrix $\frac{B}{T_{B}^{C}}$ which contains error is given by

$$
\overline{T_{B}^{C}}=\left[I-\left(\delta \boldsymbol{\theta}_{C} \times\right)\right] T_{B}^{C} .
$$

From Eq. (9), we have the following attitude error equation as

$$
\delta \dot{\boldsymbol{\theta}}_{C}=\delta \boldsymbol{\omega}_{E / C}^{C}+\delta \boldsymbol{\omega}_{I / E}^{C}+\delta \boldsymbol{\theta}_{C} \times \boldsymbol{\omega}_{I / C}^{C}+\boldsymbol{d}_{C},
$$

where $d_{C}$ denotes the gyro drift.

\subsection{Sensor Error Model}

In this work, the accelerometer bias $\boldsymbol{b}_{B}$ and gyro bias $d_{B}$ are modeled as the first order Markov processes, respectively, as follows:

$$
\begin{aligned}
& \dot{b}_{B, t}=-\frac{1}{\tau_{b}} b_{B, t}+u_{b, t}, \\
& \dot{d}_{B, t}=-\frac{1}{\tau_{d}} d_{B, t}+u_{d, t},
\end{aligned}
$$

where $\tau_{b}$ and $\tau_{d}$ are the correlation time constants and $u_{b, t}$, $u_{d, t}$ are zero mean Gaussian white noise processes.

\section{STATE AND MEASUREMENT MODELS}

\subsection{State Equation}

In order to implement the integrated navigation, here, we define the state vector. Because the double differenced carrier phases are used as the measurements in this paper, the unknown integer ambiguities should be simultaneously estimated. Therefore the state vector is defined such that it includes the INS errors as well as the integer ambiguities as follows:

$$
\begin{aligned}
& x=\left[\delta r_{C, x} \delta r_{C, y} \delta v_{C, x} \delta v_{C, y} \delta \theta_{C, x} \delta \theta_{C, y} \delta h_{C,} \delta v_{C, z} b_{B, x} b_{B, y}\right.
\end{aligned}
$$

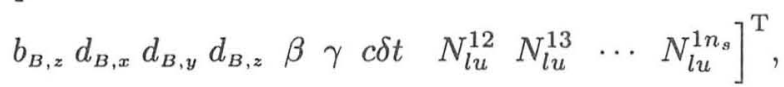

where $N_{l u}^{12}$ denotes the double differenced integer ambiguity of the satellites 1,2 and the receivers $l$, $u$, and $n_{s}$ is the number of satellites. The description of the components is listed in Table 1. The follwing dynamic model is then utilized:

$$
\dot{x}_{t}=\tilde{f}\left(x_{t}, t\right)+\eta_{t}
$$

Table 1: List of states

\begin{tabular}{|c|c|l|}
\hline \hline No. & Symbol & \multicolumn{1}{|c|}{ Error state } \\
\hline 1 & $\delta r_{x}$ & $X_{C}$-axis error in relative position \\
\hline 2 & $\delta r_{y}$ & $Y_{C}$-axis error in relative position \\
\hline 3 & $\delta v_{x}$ & $X_{C}$-axis velocity error \\
\hline 4 & $\delta v_{y}$ & $Y_{C}$-axis velocity error \\
\hline 5 & $\delta \theta_{x}$ & $X_{C}$-axis tilt error \\
\hline 6 & $\delta \theta_{y}$ & $Y_{C}$-axis tilt error \\
\hline 7 & $\gamma$ & $\sin \delta \alpha$ \\
\hline 8 & $\beta$ & $\cos \delta \alpha-1$ \\
\hline 9 & $\delta h$ & $Z_{C}$-axis altitude error \\
\hline 10 & $\delta v_{z}$ & $Z_{C}$-axis velocity error \\
\hline 11 & $b_{x}$ & $X_{B}$-axis accelerometer bias \\
\hline 12 & $b_{y}$ & $Y_{B}$-axis accelerometer bias \\
\hline 13 & $b_{z}$ & $Z_{B}$-axis accelerometer bias \\
\hline 14 & $d_{x}$ & $X_{B}$-axis gyro bias \\
\hline 15 & $d_{y}$ & $Y_{B}$-axis gyro bias \\
\hline 16 & $d_{z}$ & $Z_{B}$-axis gyro bias \\
\hline 17 & $N_{l u}^{12}$ & double differenced ambiguity \\
$\vdots$ & $\vdots$ & $\vdots$ \\
\hline \hline
\end{tabular}

where $\tilde{f}(\bullet, t)$ is the time-varying nonlinear function defined by Eqs. (6), (8), (10) and (11), and the process noise $\eta_{t}$ is assumed to be mutually independent zero mean Gaussian white noise.

By discretizing the state equation (12), we have

$$
\begin{aligned}
\boldsymbol{x}_{k+1} & =\boldsymbol{x}_{k}+\tilde{f}\left(\boldsymbol{x}_{k}, k\right) \Delta t+\boldsymbol{w}_{k} \\
& =f\left(\boldsymbol{x}_{k}, k\right)+\boldsymbol{w}_{k},
\end{aligned}
$$

where $w_{k}$ is assumed to be Gaussian white noise with zero mean and diagonal covariance matrix $Q_{k}$, and $\Delta t$ is a sampling interval of the measurement data.

\subsection{Measurement Equation}

In this paper, the measurements are the double differenced carrier phases and Doppler data. By ignoring some errors in the carrier phase data such as the remaining ionospheric and tropospheric delays and multipath errors, then the double differenced carrier phase measurement can be simply modeled by

$$
y_{k}=h\left(r_{E}\right)+\lambda N_{l u}+\varepsilon_{g, k},
$$

where $r_{E}$ is the position vector in the $E$ frame, the function $h$ is the nonlinear function that describes the distance between satellites and receivers, $N_{l u}$ is the ambiguity vector, $\lambda$ is the wave length, and $\varepsilon_{g}$ is the measurement noise.

By linearlizing (14) with the first order Taylor series approximation around the position indicated by INS, $r_{E, k}^{i}$, and applying appropriate transformations of the coordinate systems, we obtain the measurement equation of the INS posi- 
tion error in the $C$ frame as follows.

$$
\begin{aligned}
\tilde{\boldsymbol{y}}_{k} & \equiv \boldsymbol{y}_{k}-\boldsymbol{h}\left(\boldsymbol{r}_{E, k}^{i}\right) \\
& =\hat{H}_{k} \delta r_{C, k}+\lambda \boldsymbol{N}_{l u}+\boldsymbol{\varepsilon}_{g, k},
\end{aligned}
$$

where $\hat{H}_{k} \equiv-\tilde{H}_{k} T_{L, k}^{E} T_{A, k} T_{B, k} T_{C, k}^{L}$, and

$$
\begin{aligned}
\tilde{H}_{k} & \equiv\left[\frac{\partial h\left(r_{E, k}\right)}{\partial r_{E, k}}\right]_{r_{E, k}=r_{E, k}^{i}}, \\
T_{A} & \equiv\left[\begin{array}{ccc}
-\left(R_{p}+h\right) & 0 & 0 \\
0 & \frac{R_{p}+h}{\cos \lambda} & 0 \\
0 & 0 & 1
\end{array}\right], T_{B} \equiv\left[\begin{array}{lll}
0 & 1 & 0 \\
1 & 0 & 0 \\
0 & 0 & 1
\end{array}\right],
\end{aligned}
$$

where $R_{p}$ is the earth radius.

The Doppler measurement can be modeled by the change of the distance between the receiver and the satellite in the sampling interval $\Delta t$ [4]. By using the velocity error vector in the $C$ frame, $\delta v_{C}$, and the appropriate transformations similarly to the above derivations, the Doppler measurement can be formulated as

$$
D_{u, k}=G_{u, k}\left\{\tilde{T}_{C}^{E}\left(\boldsymbol{v}_{C}^{i}-\delta \boldsymbol{v}_{C}\right)-\boldsymbol{v}_{E}^{p}\right\}+1 c \dot{\delta}_{u}+\varepsilon_{d, k},
$$

where

$$
\begin{aligned}
G_{u} & \equiv\left[\boldsymbol{g}_{u}^{1^{\mathrm{T}}} \boldsymbol{g}_{u}^{2 \mathrm{~T}} \cdots \boldsymbol{g}_{u}^{n_{s} \mathrm{~T}}\right]^{\mathrm{T}}, \boldsymbol{g}_{u}^{p} \equiv\left[\frac{\partial r_{u}^{p}}{\partial \boldsymbol{r}_{E}}\right]_{\boldsymbol{r}_{E}=\boldsymbol{r}_{E}^{i}}^{\mathrm{T}}, \\
\tilde{T}_{C}^{E} & \equiv\left[\left(T_{C}^{E}\right)^{\mathrm{T}}\left(T_{C}^{E}\right)^{\mathrm{T}} \cdots\left(T_{C}^{E}\right)^{\mathrm{T}}\right]^{\mathrm{T}},
\end{aligned}
$$

and $r_{u}^{p}$ denotes the distance between the receiver $u$ and the satellite $p, v_{E}^{p}$ is the velocity of the satellite $p$ in the $E$ frame and $\varepsilon_{d}$ is the measurement noise. Then, we have the following Doppler measurement equation.

$$
\begin{aligned}
\tilde{D}_{u, k} & \equiv D_{u}+G_{u} v_{E}^{p}-G_{u} \tilde{T}_{C}^{E} v_{c}^{i} \\
& =\left[\begin{array}{ll}
-G_{u} \tilde{T}_{C}^{E} & 1
\end{array}\right]\left[\begin{array}{c}
\delta v_{C} \\
c \dot{\delta} t_{u}
\end{array}\right]+\varepsilon_{d} .
\end{aligned}
$$

Finally, from (15) and (16), we have the measurement equation for the integrated navigation in general form:

$$
z_{k}=H_{k} x_{k}+\varepsilon_{k},
$$

where $z_{k} \equiv\left[\begin{array}{ll}\tilde{y}_{k}^{\mathrm{T}} & \tilde{D}_{u, k}^{\mathrm{T}}\end{array}\right]^{\mathrm{T}}$.

\section{NONLINEAR FILTERING}

Nonlinear filtering techniques are applied to the integrated INS/GPS system in order to estimate the state vector (the errors of INS described above). In this section, firstly, we briefly review the filter algorithms of the GSF and the UKF. Then the modified Gaussian Sum filter (MGSF) algorithm is derived.

\subsection{Gaussian Sum Filter}

In the GSF, a posteriori probability density $p\left(x_{k} \mid \mathcal{Z}_{k}\right)$ is formed by the convex combination of the outputs of several EKF processed in parallel.

The priori density $p\left(x_{k} \mid \mathcal{Z}_{k-1}\right)$ is assumed that it is formulated by the sum of several normal distributions as follows:

$$
p\left(x_{k} \mid \mathcal{Z}_{k-1}\right)=\sum_{j=1}^{N} \gamma_{k}^{j^{\prime}} \mathcal{N}\left[\boldsymbol{\mu}_{k \mid k-1}^{j}, P_{\mu, k \mid k-1}^{j}\right],
$$

where $N$ is the number of distributions, and $\gamma^{j}$ is the weight for the $j$-th distribution such that $\sum_{j=1}^{N} \gamma_{k}^{j^{\prime}}=1, \gamma_{k}^{j^{\prime}} \geq 0$. And $\mathcal{N}[\theta, \Sigma]$ denotes the normal probability density function with mean $\theta$ and covariance matrix $\Sigma$. Then, by the Bayesian recursion relations, a posteriori density can be written as

$$
p\left(x_{k} \mid \mathcal{Z}_{k}\right)=\sum_{j=1}^{N} \gamma_{k}^{j} \mathcal{N}\left[\boldsymbol{\mu}_{k \mid k}^{j}, P_{\mu, k \mid k}^{j}\right],
$$

where

$$
\begin{aligned}
\mu_{k \mid k}^{j} & =\mu_{k \mid k-1}^{j}+K_{\mu, k}^{j}\left(z_{k}-H_{k} \mu_{k \mid k-1}^{j}\right), \\
P_{\mu, k \mid k}^{j} & =P_{\mu, k \mid k-1}^{j}-K_{\mu, k}^{j} H_{k} P_{\mu, k \mid k-1}^{j}, \\
K_{\mu, k}^{j} & =P_{\mu, k \mid k-1}^{j} H_{k}{ }^{\mathrm{T}}\left[H_{k} P_{\mu, k \mid k-1}^{j} H_{k}^{\mathrm{T}}+R_{k}\right]^{-1},
\end{aligned}
$$

and the weight $\gamma_{k}^{j}$ is given by

$$
\gamma_{k}^{i}=\frac{\gamma_{k}^{i^{\prime}} \beta_{k}^{i}}{\sum_{j=1}^{N} \gamma_{k}^{j^{\prime}} \beta_{k}^{j}},
$$

where

$$
\gamma_{k}^{i^{\prime}}=\gamma_{k-1}^{i}, \quad \beta_{k}^{i}=\mathcal{N}\left[\boldsymbol{z}_{k}^{g}-H_{k} \boldsymbol{\mu}_{k}^{i^{\prime}}, H_{k} P_{k}^{i^{\prime}} H_{k}^{\mathrm{T}}+R_{k}\right] .
$$

Therefore, we have the filtered estimator

$$
\hat{\boldsymbol{x}}_{k \mid k}=\sum_{j=1}^{N} \gamma_{k}^{j} \boldsymbol{\mu}_{k \mid k}^{j} .
$$

Next, the predictive prior density $p\left(x_{k+1} \mid \mathcal{Z}_{k}\right)$ can be rewritten with the same algorithm of Extended Kalman filter.

\subsection{Unscented Kalman Filter}

In the UKF, the predicted estimate $\hat{x}_{k+1 \mid k}$ and covariance $P_{k+1 \mid k}$ are calculated from a set of samples which is called the sigma points. This method is called the Unscented Transformation (UT).

Step 1 choose the sigma points $\chi_{j, k \mid k}$ which is associated with the $n$-dimensional state vector $\boldsymbol{x}_{k}$ as follows

$$
\begin{aligned}
& \chi_{0, k \mid k}=\hat{x}_{k \mid k}, \quad W_{0}=\frac{\kappa}{n+\kappa}, \\
& \chi_{j, k \mid k}=\hat{x}_{k \mid k}+\left(\sqrt{(n+\kappa) P_{k \mid k}}\right)_{j}, \\
& \chi_{j+n, k \mid k}=\hat{x}_{k \mid k}-\left(\sqrt{(n+\kappa) P_{k \mid k}}\right)_{j}, \\
& W_{j}=W_{j+n}=\frac{1}{2(n+\kappa)}, \quad(j=1,2, \ldots, n)
\end{aligned}
$$


Step2 compute a set of transformed samples through the process model (13),

$$
\chi_{j, k+1 \mid k}=f\left(\chi_{j, k \mid k}, k\right) .
$$

Step3 compute the predicting estimate and covariance as follows

$$
\begin{aligned}
\hat{x}_{k+1 \mid k} & =\sum_{j=0}^{2 n} W_{j} \chi_{j, k+1 \mid k}, \\
P_{k+1 \mid k} & =\sum_{j=0}^{2 n} W_{j} \tilde{\chi}_{j} \tilde{\chi}_{j}^{\mathrm{T}}+Q_{k},
\end{aligned}
$$

where $\tilde{\chi}_{j} \equiv \chi_{j, k+1 \mid k}-\hat{x}_{k+1 \mid k}$.

$W_{j}$ is the weight of the $j$-th point and $\kappa$ is a scaling parameter. Then, once the observation $\boldsymbol{z}(k+1)$ is obtained, $\hat{\boldsymbol{x}}_{k+1 \mid k}$ and $P_{k+1 \mid k}$ are updated to $\hat{\boldsymbol{x}}(k+1 \mid k+1)$ and $P(k+1 \mid k+1)$ as follows.

$$
\begin{aligned}
& \mathcal{Z}_{j, k+1 \mid k}=H_{k} \chi_{j, k+1 \mid k}, \\
& \hat{z}_{k+1 \mid k}=\sum_{j=0}^{2 n} W_{j} \mathcal{Z}_{j, k+1 \mid k}, \\
& P_{\nu \nu, k+1 \mid k}=\sum_{j=0}^{2 n} W_{j} \tilde{\mathcal{Z}}_{j} \tilde{\mathcal{Z}}_{j}^{\mathrm{T}}+R_{k}, \\
& P_{x \nu, k+1 \mid k}=\sum_{j=0}^{2 n} W_{j} \tilde{\boldsymbol{\chi}}_{j} \tilde{\mathcal{Z}}_{j}^{\mathrm{T}}, \\
& K_{k+1}=P_{x \nu, k+1 \mid k} P_{\nu \nu, k+1 \mid k}^{-1}, \\
& \hat{\boldsymbol{x}}_{k+1 \mid k+1}=\hat{x}_{k+1 \mid k}+K_{k}\left(z_{k}-\hat{\boldsymbol{z}}_{k+1 \mid k}\right), \\
& P_{k+1 \mid k+1}=P_{k+1 \mid k}-K_{k} P_{\nu \nu, k+1 \mid k} K_{k}^{\mathrm{T}},
\end{aligned}
$$

where $\tilde{\mathcal{Z}}_{j} \equiv \mathcal{Z}_{j, k+1 \mid k}-\hat{z}_{k+1 \mid k}$.

Since the measurement equation (17) is linear in this navigation problem, above equations (22)-(24) can be simply expressed as:

$$
\begin{aligned}
\hat{z}_{k+1 \mid k} & =H_{k} \hat{\boldsymbol{x}}_{k+1 \mid k}, \\
P_{\nu \nu, k+1 \mid k} & =H_{k} P_{k+1 \mid k} H_{k}^{\mathrm{T}}+R, \\
P_{x \nu, k+1 \mid k} & =P_{k+1 \mid k} H_{k}^{\mathrm{T}} .
\end{aligned}
$$

\subsection{Modified Gaussian Sum Filter}

In this paper, we propose the modified Gaussian Sum filter by applying the unscented transformation algorithm to the time updating algorithm of the GSF.

Step 1 similarly to the Step 1 of the UKF, for $j$-th density $\mathcal{N}\left[\boldsymbol{\mu}_{k \mid k}^{j}, P_{\mu, k \mid k}^{j}\right],(j=1,2, \ldots, N)$ in (19) for GSF, choose the sigma points and weights as follows.

$$
\begin{aligned}
& \chi_{0, k \mid k}^{j}=\mu_{k \mid k}^{j}, \quad W_{0}=\frac{\kappa}{n+\kappa}, \\
& \chi_{l, k \mid k}^{j}=\mu_{k \mid k}^{j}+\left(\sqrt{(n+\kappa) P_{\mu, k \mid k}^{j}}\right)_{l}, \\
& \chi_{l+n, k \mid k}^{j}=\mu_{k \mid k}^{j}-\left(\sqrt{(n+\kappa) P_{\mu, k \mid k}^{j}}\right)_{l}, \\
& W_{l}=W_{l+n}=\frac{1}{2(n+\kappa)}, \quad(l=1,2, \ldots, n) .
\end{aligned}
$$

Step 2 compute a set of transformed samples through the process model in (13),

$$
\chi_{l, k+1 \mid k}^{j}=\boldsymbol{f}\left(\chi_{l, k \mid k}^{j}, k\right) .
$$

Step 3 compute the $j$-th predicting estimate and covariance as follows

$$
\begin{aligned}
& \mu_{k+1 \mid k}^{j}=\sum_{l=0}^{2 n} W_{l} \chi_{l, k+1 \mid k}^{j}, \\
& P_{\mu, k+1 \mid k}^{j}=\sum_{l=0}^{2 n} W_{l} \tilde{\chi}_{l}^{j}\left(\tilde{\chi}_{l}^{j}\right)^{\mathrm{T}}+Q_{k},
\end{aligned}
$$

where $\tilde{\chi}_{l}^{j} \equiv \chi_{l, k+1 \mid k}^{j}-\mu_{k+1 \mid k}^{j}$.

In the MGSF, the original time updating algorithm are substituted by (32) and (33) respectively.

\section{EXPERIMENTAL RESULTS}

The experiments of the INS/GPS In-Motion Alignment and navigation algorithms described above were carried out by using simulated INS and GPS data. In the experiments, we assume the vehicle runs at a speed of around $15[\mathrm{~km} / \mathrm{h}]$ for about 10 minutes. The speed at the start point was $0[\mathrm{~km} / \mathrm{h}]$, and the test trajectory in the local level horizontal plane is shown in Fig. 3. The data were obtained by utilizing the Matlab 6.5 and INS Toolbox 1.0 (GPSoft LLC.) at 50 [Hz] rate for IMU and at $1[\mathrm{~Hz}]$ rate for GPS.

In this simulation we assume the system components consist of NovAtel GPS receiver OEM4 and Tamagawa Seiki TA7421 strapdown INS. The IMU errors and the measurement noise of GPS are generated based on the equipment specifications shown in Table 2

Four types of filters, i.e. EKF, GSF, UKF and MGSF are used in the experiments and compared. The nonlinearity of the INS usually occurs when there exist large attitude errors. Hence the experiments are conducted by intentionally adding -60 [deg.] of the initial azimuth error, and we assume that there exist no errors in the other initial values. In the EKF and UKF, the initial estimate $\hat{x}_{0 \mid-1}=0$, and $P_{0 \mid-1}$ and $Q$ are configured from the nominal equipment specifications in Table 2. 


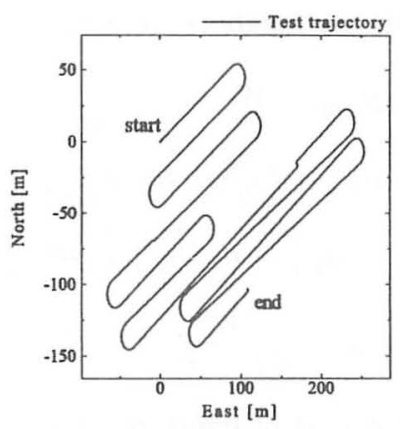

Fig. 3: Test trajectory

Table 2: Sensor error specification of IMU

\begin{tabular}{|c|cl|}
\hline \hline Accelerometer & & \\
\hline Bias & 80 & {$[\mu \mathrm{G}](1 \sigma)$} \\
Scale factor & 150 & {$[\mathrm{ppm}](1 \sigma)$} \\
Random error & 0.0003 & {$[\mathrm{~m} / \mathrm{s}]^{2}$} \\
\hline \hline (Ring Laser) Gyro & & \\
\hline Bias & 20 & {$[\mathrm{deg} / \mathrm{h}](1 \sigma)$} \\
Scale factor & 500 & {$[\mathrm{ppm}](1 \sigma)$} \\
Random error & 0.06 & {$[\mathrm{deg} / \sqrt{\mathrm{h}}]$} \\
\hline \hline
\end{tabular}

In the GSF and MGSF, three normal distributions are utilized, i.e. $N=3$, and $P_{\mu, 0 \mid-1}^{j}=P_{0 \mid-1}, j=1,2,3$. The initial estimates $\boldsymbol{\mu}_{0 \mid-1}^{j}=0, j=1,2,3$ except for the 7th and 8-th components of the state vector (see Table 1), $\beta$ and $\gamma$, that represent the azimuth error. They are assumed to have the initial azimuth error estimates such that $\delta \alpha=-60,0,+60$ [deg.]. Also in the UKF and MGSF, $n$ in (21) and (31) is dimension, and we assumed $n+\kappa=3$.
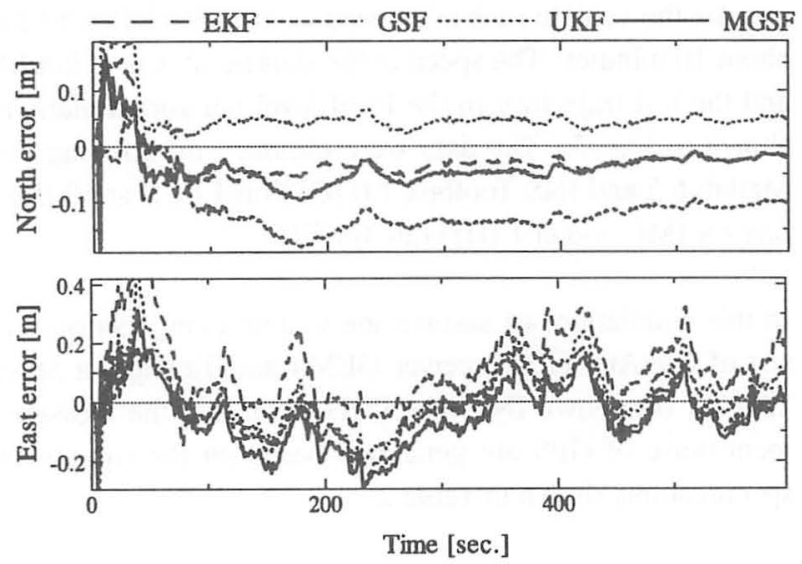

Fig. 4: Positioning errors

The processing results are shown in Figs. 4 and 5. Fig. 4 shows the results of the positioning and comparison of the positioning errors. From Fig. 4 we can see that the MGSF show better performance than GSF, EKF and UKF.

Finally, Fig. 5 shows the results of the azimuth errors. From

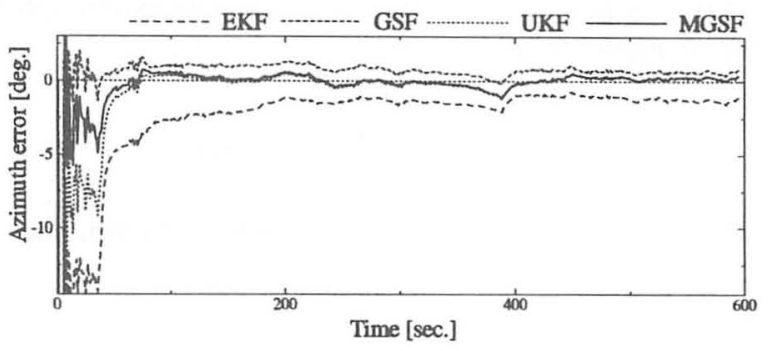

Fig. 5: Azimuth errors

Fig. 5, we can see that MGSF and GSF show faster convergence than EKF and UKF, and MGSF and UKF show better performances than EKF and GSF after 80 [sec].

\section{CONCLUSION}

In this paper, the modified Gaussian Sum filtering algorithm was derived by applying the unscented transformation to the Gaussian Sum filter, and it was applied to the GPS/INS integrated system. The algorithm was tested and compared with the EKF, GSF and UKF by using simulated data. The experimental results show the derived MGSF has an ability to outperform the other filters under large azimuth errors. In the future study, we plan to test the algorithms by using real IMU and GPS data.

\section{REFERENCES}

[1] R. M. Rogers: Applied Mathematics in Integrated Navigation Systems, 2nd edition, AIAA, Virginia, 2003.

[2] S. Fujioka, M. Tanikawara, M. Nishiyama, Y. Kubo and S. Sugimoto: Comparison of Nonlinear Filtering Methods for INS/GPS In-Motion Alignment, Proc. of the Institute of Navigation, ION GNSS 2005, pp. 467-477, Long Beach, CA, 2005.

[3] M. Nishiyama, S. Fujioka, Y. Kubo, T. Sato and S. Sugimoto: Performance Studies of Nonlinear Filtering Methods in INS/GPS In-Motion Alignment, Proc. 19th Int. Tech. Meeting of the Satellite Division of The Institute of Navigation (ION GNSS 2006), pp. 2733-2742, Fort Worth, TX, Sep., 2006.

[4] P. Misra and P. Enge: Global Positioning System - Signals, Measurements, and Performance, Ganga-Jamuna Press, Massachusetts, 2001. 